5-1999

\title{
A Worst-Case Analysis of Direct-Sequence Spread-Spectrum in Multipath Channels
}

\author{
Murad Hizlan \\ Cleveland State University, hizlan@cxvax.csuohio.edu \\ Xuedong Liu

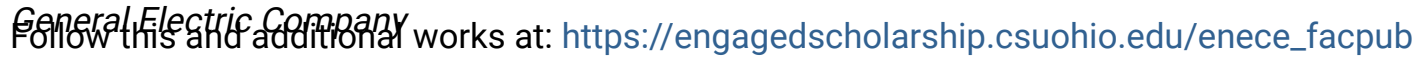 \\ Part of the Systems and Communications Commons
}

How does access to this work benefit you? Let us know!

\section{Publisher's Statement}

NOTICE: this is the author's version of a work that was accepted for publication in Journal of the Franklin Institute-Engineering and Applied Mathematics. Changes resulting from the publishing process, such as peer review, editing, corrections, structural formatting, and other quality control mechanisms may not be reflected in this document. Changes may have been made to this work since it was submitted for publication. A definitive version was subsequently published in Journal of the Franklin Institute-Engineering and Applied Mathematics, 336, 4, (05-01-1999); 10.1016/S0016-0032(97)00078-1

\section{Original Citation}

Hizlan, M., , \& Liu, X. (1999). A worst-case analysis of direct-sequence spread-spectrum in multipath channels. Journal of The Franklin Institute, 336(4), 611-625.

\section{Repository Citation}

Hizlan, Murad and Liu, Xuedong, "A Worst-Case Analysis of Direct-Sequence Spread-Spectrum in Multipath Channels" (1999). Electrical Engineering \& Computer Science Faculty Publications. 72.

https://engagedscholarship.csuohio.edu/enece_facpub/72

This Article is brought to you for free and open access by the Electrical Engineering \& Computer Science Department at EngagedScholarship@CSU. It has been accepted for inclusion in Electrical Engineering \& Computer Science Faculty Publications by an authorized administrator of EngagedScholarship@CSU. For more information, please contact library.es@csuohio.edu. 


\title{
A worst-case analysis of direct-sequence spread- spectrum in multipath channels
}

\author{
Murad Hizlan ${ }^{\mathrm{a}, *}$, Xuedong Liu ${ }^{\mathrm{b}}$ \\ ${ }^{a}$ Department of Electrical and Computer Engineering, Cleveland State University, Cleveland, $\mathrm{OH} 44115$, \\ U.S.A. \\ ${ }^{\mathrm{b}}$ General Electric Company, Fort Wayne, IN 4680I, U.S.A.
}

\section{Introduction}

Consider a communication situation where, in general, $K$ transmitters using a pseudorandom direct-sequence spread-spectrum multiple-access (DS/SSMA) scheme share an indoors environment characterized by multipath interference with $L$ discrete paths from each transmitter, and additive white Gaussian noise (AWGN). Although we use the indoors environment as an example, and consequently assume a Rayleightype fading, our methods are applicable to any fading situation where the received signal can be modeled as resulting from a number of discrete paths.

Many aspects of SSMA and indoors environment, such as multiuser average error

* Corresponding author. 
probabilities and propagation measurements have been considered in the literature [1-6]. Worst-case performance measures, however, have received much less attention compared with the work on the average probability of error. One reason for the lack of attention on the worst case is the assumption that the worst-case performance will be so poor that it will not be of any practical or theoretical use. Indeed, commonly used system models and signal-to-noise ratio definitions immediately point in this direction. However, through a judicious definition of signal-to-interference ratio, it is possible, as we show in this paper, to obtain sensible results for the worst-case performance.

Our definition of the worst-case performance allows us to evaluate system performance regardless of any particular statistical distribution. We use two signal-tonoise ratio expressions, one for the AWGN and the other for the interference from all the paths of all the transmitters. We obtain, for a special case, an easy-to-evaluate upper bound on the worst-case error probability that provides an overall picture of the worst possible performance of the system with any given signal-to-noise and signal-to-interference ratio.

The rest of this paper is organized as follows. In Section 2, we present precise definitions of the parameters and performance measure of interest for the system under consideration. In Section 3, we derive an upper bound on the worst-case error probability of the system for a special case. We conclude, in Section 4, with numerical examples and a comparison of the worst-case multipath interference with the worstcase multiuser interference.

\section{System model}

We consider a DS/SSMA BPSK system operating in an indoor wireless channel. We assume that the multiple access system comprises $K$ transmitters, labeled by integers 1 through $K$, as shown in Fig. 1. The data waveform from transmitter $k$ can be expressed as

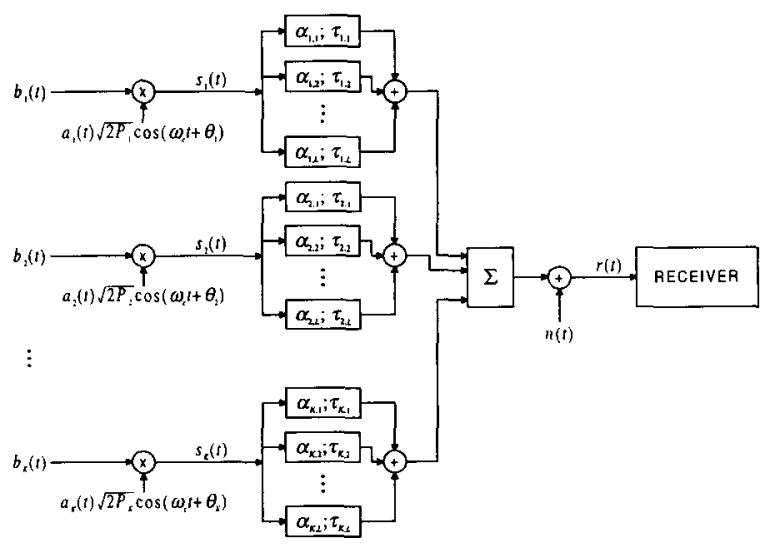

Fig. 1. DS/SSMA BPSK multipath system model. 


$$
b_{k}(t)=\sum_{i=-\infty}^{+\infty} b_{k, i} P_{T}(t-i T),
$$

where $T$ is the data bit duration, $b_{k, i}$ is the $i$ th bit from the $k$ th transmitter and takes on values +1 and -1 with equal probability, and

$$
P_{\mathrm{T}}(\mathrm{t})= \begin{cases}1, & \text { if } 0 \leqslant t<T, \\ 0, & \text { otherwise }\end{cases}
$$

Each transmitter has a spreading code sequence $a_{k, j}$, which determines the chip waveform

$$
a_{k}(t)=\sum_{j=-\infty}^{+\infty} a_{k, j} \psi\left(t-j T_{\mathrm{c}}\right),
$$

where $T_{\mathrm{c}} \triangleq T / N$ is the chip duration, $N$ is the number of chips per bit, $\psi(t)$ is the basic pulse shape with unit power, so that

$$
\frac{1}{T_{\mathrm{c}}} \int_{0}^{T_{\mathrm{c}}} \psi^{2}(t) \mathrm{d} t=1
$$

and $a_{k, j}$ is the $j$ th chip from the $k$ th transmitter and is assumed to form a sequence of i.i.d. Bernoulli random variables that take on values +1 and -1 with equal probability. Hence, the signal from transmitter $k$ has the form [7]

$$
s_{k}(t)=\sqrt{2 P_{k}} a_{k}(t) b_{k}(t) \cos \left(w_{0} t+\theta_{k}\right),
$$

where $P_{k}$ is the power and $\theta_{k}$ is phase angle of transmitter $k$, and $\omega_{0}$ is the carrier frequency with $\omega_{0} \gg T^{-1}$.

It is well known that the indoors wireless channel is characterized as a multipath fading channel due to the presence of many reflectors and scatterers in the environment. In the case of spread-spectrum transmission, the spread bandwidth of the signal exceeds the coherence bandwidth of the channel, and thus the multipath components can be resolved into a discrete number of Rayleigh-distributed paths [8]. The exact number of paths is a function of the multipath spread and the spread-spectrum bandwidth.

We therefore assume that the received signal in the indoors environment will be due to a number of discrete paths, say $L$ paths, each with a different attenuation $\alpha$, time delay $\tau$, and RF phase $\theta$. With this, we can express the received signal from transmitter $k$ as

$$
r_{k}(t)=\sum_{l=1}^{L} \alpha_{k, l} \sqrt{2 P_{k}} a_{k}\left(t-\tau_{k, l}\right) b_{k}\left(t-\tau_{k, l}\right) \cos \left[\omega_{0}\left(t-\tau_{k, l}\right)+\theta_{k, l}\right],
$$

where $\alpha_{k, l}$ is the Rayleigh distributed path attenuation, $\tau_{k, l}$ is the time delay, and $\theta_{k, l}$ is the phase delay of path $l$ from transmitter $k$. Here, $\tau_{k . l}$ is uniformly distributed in $[0, T)$ and $\theta_{k, l}$ is uniformly distributed in $[0,2 \pi)$. Hence, the total received signal from all the transmitters in the presence of AWGN is given by 


$$
r(t)=\sum_{k=1}^{K} \sum_{l=1}^{L} \alpha_{k, l} \sqrt{2 P_{k}} a_{k}\left(t-\tau_{k, l}\right) b\left(t-\tau_{k, l}\right) \cos \left(\omega_{0} t+\phi_{k, l}\right)+n(t),
$$

where $n(t)$ is a Gaussian process modeling AWGN with double-sided power spectral density of $N_{0} / 2$, and $\phi_{k, l} \triangleq \theta_{k, l}-\omega_{0} \tau_{k, l}$ is uniformly distributed in $[0,2 \pi)$.

Without loss of generality, we restrict our attention to the detection of bit 0 from transmitter 1 . The non-diversity receiver is assumed to be in phase and time lock with one of the paths from transmitter 1, say path 1 . Hence, we can set $\tau_{1,1}=0$ and $\phi_{1,1}=0$. A correlation receiver matched to the signal for bit 0 from this path produces the output

$$
\begin{aligned}
z(T)= & \int_{0}^{T} r(t) a_{1}(t) \cos \left(\omega_{0} t\right) \mathrm{d} t \\
= & \alpha_{1,1} b_{1,0} \sqrt{P_{1} / 2} T+\eta+\sum_{l=2}^{L} \alpha_{1, l} \sqrt{P_{1} / 2} \cos \left(\phi_{1, l}\right) \int_{0}^{T} a_{1}(t) a_{1}\left(t-\tau_{1, l}\right) b_{1}\left(t-\tau_{1, l}\right) \mathrm{d} t \\
& +\sum_{k=2}^{K} \sum_{l=1}^{L} \alpha_{k, l} \sqrt{P_{\mathbf{k}} / 2} \cos \left(\phi_{k, l}\right) \int_{0}^{T} a_{1}(t) a_{k}\left(t-\tau_{k, l}\right) b_{k}\left(t-\tau_{k, l}\right) \mathrm{d} t
\end{aligned}
$$

where double frequency components are ignored since $\omega_{0} \gg T^{-1}$. Here, $\eta$ is a zeromean Gaussian random variable with variance $N_{0} T / 4$.

We show in [9] that the detector output (8) can be rewritten as

$$
\begin{aligned}
z(T)=\alpha_{1,1} b_{1,0} \sqrt{P_{1} / 2} T+ & +\sum_{l=2}^{L} \alpha_{1, l} \sqrt{P_{1} / 2} T_{\mathrm{c}} \cos \left(\phi_{1, l}\right)\left[\bar{\Psi}_{\delta_{1, l}} X_{1, l}+\Psi_{\delta_{1, l}} Y_{1, l}\right] \\
+ & \sum_{k=2}^{K} \sum_{l=1}^{L} \alpha_{k, l} \sqrt{P_{k} / 2} T_{\mathrm{c}} \cos \left(\phi_{k, l}\right)\left[\bar{\Psi}_{\delta_{k, l}} X_{k, l}+\Psi_{\delta_{k, l}} Y_{k, l}\right]
\end{aligned}
$$

where we define the two partial correlation functions as

$$
\begin{aligned}
& \Psi_{\delta_{k, l}} \triangleq \frac{1}{T_{\mathrm{c}}} \int_{0}^{\delta_{k, l}} \psi(t) \psi\left(t-\delta_{k, l}+T_{\mathrm{c}}\right) \mathrm{d} t, \\
& \bar{\Psi}_{\delta_{k, l}} \triangleq \frac{1}{T_{\mathrm{c}}} \int_{\delta_{k, l}}^{T_{\mathrm{c}}} \psi(t) \psi\left(t-\delta_{k, l}\right) \mathrm{d} t,
\end{aligned}
$$

with

$$
\delta_{k, l} \triangleq \tau_{k, l}-m_{k, l} T_{\mathrm{c}}, \quad m_{k, l} \triangleq\left\lfloor\frac{\tau_{k, l}}{T_{\mathrm{c}}}\right\rfloor
$$

and $X_{k, l}, Y_{k, l}$ as

$$
X_{k, l} \triangleq b_{k,-1} \sum_{j=0}^{m_{k, l}-1} a_{1, j} a_{k, j-m_{k, l}}+b_{k, 0} \sum_{j=m_{k, l}}^{n-1} a_{1, j} a_{k, j-m_{k, l}}
$$




$$
Y_{k, l} \triangleq b_{k,-1} \sum_{j=0}^{m_{k, l}} a_{1, j} a_{k, j-m_{k, l}-1}+b_{k, 0} \sum_{j=m_{k, l}+1}^{n-1} a_{1, j} a_{k, j-m_{k, l}-1} .
$$

Note that the detector will make a wrong decision if $z(T)$ is negative when $b_{1,0}=+1$ or if $z(T)$ is positive when $b_{1,0}=-1$. As clearly seen in (9), the terms interfering with the detection of $b_{1,0}$ involve thermal noise $\eta$, and the other paths of transmitter 1 and all paths of all the other transmitters through i.i.d. random variables $b_{k, 0}, b_{k,-1}$, and $a_{k, j}$.

In the next section, we will define the worst-case performance of interest and the associated signal-to-noise ratio expressions, and consider a Chernoff-type upper bound for a special case of (9).

\section{Worst-case error probability}

We now consider the problem of analyzing the worst-case performance of the system described above when a hard decision is made on the received bit $b_{1,0}$, i.e.

$$
\hat{b}_{1,0}=\left\{\begin{aligned}
1, & \text { if } z(T) \geqslant 0 \\
-1, & \text { if } z(T)<0 .
\end{aligned}\right.
$$

We can in principle evaluate the conditional probability of error given a set of relative phase angles $\Theta \triangleq\left\{\theta_{k, l}: 1 \leqslant k \leqslant K, 1 \leqslant l \leqslant L\right\}$, time delays $\mathscr{T} \triangleq\left\{\tau_{k, l}: 1 \leq k \leqslant K\right.$, $1 \leqslant l \leqslant L\}$, fading coefficients $\mathscr{A} \triangleq\left\{\alpha_{k, l}: 1 \leqslant k \leqslant K, 1 \leqslant l \leqslant L\right\}$, and transmitter powers $\mathscr{P} \triangleq\left\{P_{k}: 1 \leqslant k \leqslant K\right\}$. Since $z(T)$ has a symmetric distribution and $b_{1,0}$ is equally likely to be \pm 1 , we have, for the conditional error probability,

$$
\begin{aligned}
P_{\mathrm{b}}(\Theta, \mathscr{T}, \mathscr{A}, \mathscr{P}) & =\operatorname{Pr}\left\{z(T)<0 \mid b_{1,0}=+1\right\} \\
& =\operatorname{Pr}\left\{z(T) \geqslant 0 \mid b_{1,0}=-1\right\} .
\end{aligned}
$$

Substituting (9) with $b_{1,0}=-1$ into Eq (14), we have

$$
\begin{aligned}
& P_{\mathrm{b}}(\Theta, \mathscr{T}, \mathscr{A}, \mathscr{P}) \\
& \quad=\operatorname{Pr}\left\{\sum_{k=1}^{K} \sum_{\substack{l=1 \\
(k=1, l \geqslant 2)}}^{L} \frac{\alpha_{k, l}}{\alpha_{1,1}} \sqrt{\frac{P_{k}}{P_{1}}} \cos \left(\phi_{\mathrm{k}, l}\right)\left[\bar{\Psi}_{\delta_{k, l}} X_{k, l}+\Psi_{\delta_{k, l}} Y_{k, l}\right] \geqslant N+\tilde{\eta}\right\},
\end{aligned}
$$

where $\tilde{\eta}$ has distribution $\mathscr{N}\left(0, N_{0} N / 2 \alpha_{1,1}^{2} P_{1} T_{\mathrm{c}}\right)$.

In this paper, we are interested in determining the worst-case value of $P_{\mathrm{b}}(\Theta, \mathscr{T}, \mathscr{A}, \mathscr{P})$ over all values of $\Theta, \mathscr{T}, \mathscr{A}$ and $\mathscr{P}$. Hence, we define the worst-case error probability as our measure of performance,

$$
\varepsilon(\sigma, \gamma) \triangleq \max _{\Theta, \mathscr{T}, \mathscr{A}, \mathscr{P}} P_{\mathrm{b}}(\Theta, \mathscr{T}, \mathscr{A}, \mathscr{P}),
$$

where $1 / \gamma^{2}$ is the signal-to-noise ratio for the AWGN, with 


$$
\frac{1}{\gamma^{2}} \triangleq \frac{\alpha_{1,1}^{2} P_{1} T}{N_{0}}=\frac{E_{\mathrm{b}}}{N_{0}}
$$

and the maximum is taken over all $\Theta \in[0,2 \pi)^{K L}, \mathscr{T} \in[0, T)^{K L}, \mathscr{A} \geqslant \overrightarrow{0}$, and $\mathscr{P} \geqslant \overrightarrow{0}$. Obviously, $\Theta, \mathscr{T}, \mathscr{A}$ and $\mathscr{P}$ are parameters that are continually changing and usually unavailable. Furthermore, if they are left unconstrained, they would result in a worstcase error probability approaching 1 . Therefore, Eq (16) is not very useful in its current form.

However, we can define a signal-to-interference ratio that incorporates all of these parameters. Observing from $\mathrm{Eq}(9)$ that the received signal power per chip is

$$
\frac{\alpha_{1,1}^{2} P_{1} T^{2}}{2 N}
$$

and the received interference power is

$$
\sum_{k=1}^{K} \sum_{(k=1, l \geqslant 2)}^{L} \frac{\alpha_{k, l}^{2} P_{k} N T_{\mathrm{c}}^{2}}{2} \cos ^{2}\left(\phi_{\mathrm{k}, 1}\right)\left[\bar{\Psi}_{\delta_{k, l}}^{2}+\Psi_{\delta_{k, l}}^{2}\right],
$$

we define the signal-to-interference ratio as the ratio of (18) to (19). With this, we can evaluate the worst-case error probability subject to a constraint on the ratio of interference power to signal power:

$$
\begin{aligned}
\varepsilon(\sigma, \gamma)= & \max _{\Theta, \mathscr{T}, \mathscr{A}, \mathscr{P}} P_{\mathrm{b}}(\Theta, \mathscr{T}, \mathscr{A}, \mathscr{P}) \text { subject to } \\
& \sum_{k=1}^{K} \sum_{\substack{l=1 \\
(k=1, l \geqslant 2)}}^{L} \frac{\alpha_{\mathrm{k}, l}^{2} P_{\mathbf{k}}}{\alpha_{1,1}^{2} P_{1}} \cos ^{2}\left(\phi_{k, l}\right)\left[\bar{\Psi}_{\delta_{k, l}}^{2}+\Psi_{\delta_{k, l}}^{2}\right] \leqslant \sigma^{2},
\end{aligned}
$$

where $\sigma^{2}$ is the constraint on interference-to-signal ratio.

We remark here that the worst-case error probability (20) can be evaluated without reference to any particular statistical distribution for the parameters involved. This makes sense because of the nature of the worst-case error probability; explicit statistical distributions would result in an average error probability. We further note that signal-to-noise ratio (17) for AWGN is defined as conditioned on $\alpha_{1,1}$. One can obtain the worst-case error probability for a particular statistical distribution on $\alpha_{1,1}$ (such as Rayleigh) by taking the expectation of (20) over the distribution of $1 / \gamma^{2}$.

Turning our attention back to the evaluation of (20), we see from (15) and (20) that the maximization problem is equivalent to

$$
\begin{gathered}
\varepsilon(\sigma, \gamma)=\max _{u_{k, l}, v_{k, l}} \operatorname{Pr}\left\{\sum_{k=1}^{K} \sum_{\substack{l=1 \\
(k=1, l \geqslant 2)}}^{L}\left[u_{k, l} X_{k, l}+v_{k, l} Y_{k, l}\right] \geqslant N+\tilde{\eta}\right\} \\
\text { subject to } \sum_{k=1}^{K} \sum_{\substack{l=1 \\
(k=1, l \geqslant 2)}}^{L}\left[u_{k, l}^{2}+v_{k, l}^{2}\right] \leqslant \sigma^{2},
\end{gathered}
$$

where we have used 


$$
u_{k, l} \triangleq \frac{\alpha_{k, l}}{\alpha_{1, l}} \sqrt{\frac{P_{k}}{P_{1}}} \cos \left(\phi_{k, l}\right) \Psi_{\delta_{k, l},} \quad v_{k, l} \triangleq \frac{\alpha_{k, l}}{\alpha_{1, l}} \sqrt{\frac{P_{k}}{P_{1}}} \cos \left(\phi_{k, l}\right) \Psi_{\delta_{k, l}}
$$

We further simplify (21) by defining

$$
\tilde{z} \triangleq \sum_{k=1}^{K} \sum_{\substack{l=1 \\(k=1, l \geqslant 2)}}^{L}\left[u_{k, l} X_{k, l}+v_{k, l} Y_{k, l}\right]
$$

and invoking the symmetry of $\tilde{\eta}$ :

$$
\varepsilon(\sigma, \gamma)=\max _{u_{k, l} k_{k, l}} \operatorname{Pr}\{\tilde{z}+\tilde{\eta} \geqslant N\} \text { subject to } \sum_{k=1}^{K} \sum_{\substack{l=1 \\(k=1, l \geqslant 2)}}^{L}\left[u_{k, l}^{2}+v_{k, l}^{2}\right] \leqslant \sigma^{2} .
$$

It is well known that, except for very simple cases, it is not possible to obtain a closed form solution for $\operatorname{Pr}\{\tilde{z}+\tilde{\eta} \geqslant N\}$. Here, we are interested in obtaining a Chernoff-type upper bound on this term. By maximizing the bound afterwards, we will obtain an upper bound on the worst-case error probability of our system. The Chernoff bound is given $[10]$ as

$$
\operatorname{Pr}\{\tilde{z}+\tilde{\eta} \geqslant N\} \leqslant \mathrm{e}^{-\lambda N} M_{\tilde{z}}(\lambda) M_{\tilde{\eta}}(\lambda),
$$

where $M_{\bar{z}}(\lambda)$ and $M_{\tilde{\eta}}(\lambda)$ are the moment generating functions of $\tilde{z}$ and $\tilde{\eta}$, respectively.

First, consider the moment generating function of $\tilde{\eta}$. Since $\tilde{\eta}$ is a Gaussian random variable with distribution $\mathscr{N}\left(0, N_{0} N / 2 \alpha_{1,1}^{2} P_{1} T_{\mathrm{c}}\right)=\mathscr{N}\left(0, N^{2} \gamma^{2} / 2\right)$, we have [10]:

$$
M_{\tilde{\eta}}(\lambda)=\exp \left[\frac{N^{2} \gamma^{2} \lambda^{2}}{4}\right] .
$$

Next, consider $M_{z}(\lambda)$. Evaluation of this moment generating function for an arbitrary number of transmitters and paths is a prohibitively difficult problem. As stated earlier, we are interested in evaluating the worst-case error probability for a special case, where we consider one transmitter with two paths so that $K=1$ and $L=2$. We discuss the merits of considering this special case in Section 4. We show in [9] that, for this special case,

$$
M_{\mathbf{z}}(\lambda)=\frac{1}{N} \cosh ^{N}(\lambda u) \cosh ^{N}(\lambda v) \frac{\left[1+\tanh ^{2}(\lambda u) \tanh ^{2}(\lambda v)\right]^{N}-1}{\tanh ^{2}(\lambda u) \tanh ^{2}(\lambda v)},
$$

where we defined $u \triangleq u_{1,2}$ and $v \triangleq v_{1,2}$.

We can now use (25) and (24) to obtain an upper bound on the worst-case error probability for the special case of $K=1$ and $L=2$ as follows:

$$
\varepsilon(\sigma, \gamma)=\max _{u, v} \operatorname{Pr}\{\tilde{z}+\tilde{\eta} \geqslant N\} \leqslant \max _{u, v} \mathrm{e}^{-\lambda N} M_{z}(\lambda) M_{\tilde{\eta}}(\lambda) \text { subject to } u^{2}+v^{2} \leqslant \sigma^{2} .
$$

Observe that the only term which is a function of $u$ or $v$ in (28) is the $M_{\bar{z}}(\lambda)$ term. Hence, we now concentrate on maximizing this term. The problem can be stated as 


$$
\begin{aligned}
\max _{u, v}\left(\frac{1}{N} \cosh ^{N}(\lambda u) \cosh ^{N}(\lambda v) \frac{\left[1+\tanh ^{2}(\lambda u) \tanh ^{2}(\lambda v)\right]^{N}-1}{\tanh ^{2}(\lambda u) \tanh ^{2}(\lambda v)}\right) \\
\text { subject to } u^{2}+v^{2} \leqslant \sigma^{2} .
\end{aligned}
$$

Let us investigate (29) term by term. First consider $\cosh ^{N}(\lambda u) \cosh ^{N}(\lambda v)$. It can be easily shown using elementary calculus that for $a^{2}+b^{2} \leqslant c^{2}$,

$$
\cosh (a) \cosh (b) \leqslant \cosh ^{2}(c / \sqrt{2})
$$

with equality when $a=b= \pm c / \sqrt{2}$. Hence, we have

$$
\max _{u, v} \cosh ^{N}(\lambda u) \cosh ^{N}(\lambda v)=\cosh ^{2 N}(\lambda \sigma / \sqrt{2}) \text { subject to } u^{2}+v^{2} \leqslant \sigma^{2} .
$$

Now consider the term

$$
\frac{\left[1+\tanh ^{2}(\lambda u) \tanh ^{2}(\lambda v)\right]^{N}-1}{\tanh ^{2}(\lambda u) \tanh ^{2}(\lambda v)}=\sum_{m=0}^{N-1}\left[1+\tanh ^{2}(\lambda u) \tanh ^{2}(\lambda v)\right]^{N-1-m} .
$$

Again, it is a matter of elementary calculus to show that for $a^{2}+b^{2} \leqslant c^{2}$, we have

$$
\tanh (a) \tanh (b) \leqslant \tanh ^{2}(\mathrm{c} / \sqrt{2})
$$

with equality when $a=b=c / \sqrt{2}$. Hence,

$$
\begin{aligned}
\max _{u, r^{\prime}} \frac{\left[1+\tanh ^{2}(\lambda u) \tanh ^{2}(\lambda v)\right]^{N}-1}{\tanh ^{2}(\lambda u) \tanh ^{2}(\lambda v)} \\
=\frac{\left[1+\tanh ^{4}(\lambda \sigma / \sqrt{2})\right]^{N}-1}{\tanh ^{4}(\lambda \sigma / \sqrt{2})} \text { subject to } u^{2}+v^{2} \leqslant \sigma^{2} .
\end{aligned}
$$

Since both terms are maximized at the same point, we can combine (29), (31) and (34) to obtain

$$
\begin{aligned}
\max _{u, t}\left(\frac{1}{N} \cosh ^{N}(\lambda u) \cosh ^{N}(\lambda v) \frac{\left[1+\tanh ^{2}(\lambda u) \tanh ^{2}(\lambda v)\right]^{N}-1}{\tanh ^{2}(\lambda u) \tanh ^{2}(\lambda v)}\right) \\
=\frac{1}{N} \cosh ^{2 N}(\lambda \sigma / \sqrt{2}) \frac{\left[1+\tanh ^{4}(\lambda \sigma / \sqrt{2})\right]^{N}-1}{\tanh ^{4}(\lambda \sigma / \sqrt{2})} \text { subject to } u^{2}+v^{2} \leqslant \sigma^{2} .
\end{aligned}
$$

We can now express the upper bound on the worst-case error probability as

$$
\begin{aligned}
\varepsilon(\sigma, \gamma) \leqslant \max _{u, i} \mathrm{e}^{-\lambda N} M_{z}(\lambda) M_{\tilde{\eta}}(\lambda) \\
=\frac{1}{N} \cosh ^{2 N}(\hat{\lambda} \sigma / \sqrt{2}) \frac{\left[1+\tanh ^{4}(\lambda \sigma / \sqrt{2})\right]^{N}-1}{\tanh ^{4}(\lambda \sigma / \sqrt{2})} \mathrm{e}^{\lambda N\left(\lambda N \gamma^{2} / 4-1\right)}
\end{aligned}
$$

Since (35) holds true for all $\lambda \geqslant 0$, we can tighten this bound through a numerical minimization over $\lambda$ to get the main result of this section: 


$$
\varepsilon(\sigma, \gamma) \leqslant \min _{i \geqslant 0} \frac{1}{N} \cosh ^{2 N}(\lambda \sigma / \sqrt{2}) \frac{\left[1+\tanh ^{4}(\lambda \sigma / \sqrt{2})\right]^{N}-1}{\tanh ^{4}(\lambda \sigma / \sqrt{2})} \mathrm{e}^{\lambda N\left(\lambda N_{i}^{2} / 4-1\right)} .
$$

In the next section, we will present several numerical examples of the bound (36), as well as a similar bound for non-multipath direct-sequence multiple access. We will conclude with observations and comparisons.

\section{Discussion and conclusions}

\subsection{The special case}

In the previous section, we have developed a Chernoff-type upper bound on the worst-case error probability of a multiaccess direct-sequence spread-spectrum system operating in a multipath environment, and derived a closed form expression for this bound for the special case of one transmitter with two paths.

As stated before, the interference in the general system is due to three components: (1) additive white Gaussian noise, (2) multipath interference from all the paths, except the one being detected, of transmitter 1, and (3) multipath interference from all the paths of all the other transmitters in the multiaccess network.

It should be clear, for Case 3 above, that whether the other transmitters' signals arrive through multiple paths or through one path each does not make any difference in terms of the basic nature of that interference component. This is because interference due to all paths from all the other transmitters is independent of the signal received from transmitter 1 . Hence, having $K-1$ additional transmitters with $L$ paths each is equivalent to having $(K-1) L$ additional transmitters with one path each.

Furthermore, it should also be clear that the interference for Case 2, that from the other paths of transmitter 1, is substantially different from the interference for Case 3. This is because interference from other paths of transmitter 1 is not independent of the signal received from transmitter 1 .

Therefore, by considering a special case of the problem with $K=1$ and $L=2$, we are ignoring two terms: multiaccess interference and interference due to more than one path. However, we are still capturing the essence of the problem, that is, the worst-case performance due to an interference term which is not independent of the signal.

It can be expected that considering more than two paths from transmitter 1 will simply cause an increase in the level of interference; it will not change the basic form of that interference. Since we are interested in the worst-case performance with a constraint on the interference power, the results for $K=1$ with arbitrary $L$ will be very similar to those for $K=1$ and $L=2$.

Considering more than one transmitter, on the other hand, will introduce a different type of interference, that of independent (from transmitter 1) multiaccess terms. We investigate this situation separately to get a basic understanding of the nature of this interference.

In [11], Hizlan and Hughes have obtained a Chernoff-type upper bound on the 
worst-case error probability of multiaccess direct-sequence spread-spectrum with a constraint on the interference power. This happens to be a special case of our model with $L=1$, i.e. no multipath interference. The result in that case is given [11] as

$$
\begin{aligned}
\varepsilon(\sigma, \gamma) \leqslant \min _{\lambda \geqslant 0} 2^{1-N} \cosh ^{2 K-2}\left(\frac{\lambda \sigma}{\sqrt{2 K-2}}\right) \\
\times\left[\cosh ^{K-1}\left(\frac{2 \lambda \sigma}{\sqrt{2 K-2}}\right)+1\right]^{N-1} \mathrm{e}^{\lambda N\left(\lambda N \gamma^{2} / 4-1\right)} .
\end{aligned}
$$

We use (37) to get an understanding of the nature of interference type 3 discussed above.

\subsection{Effects of multipath interference}

We plot several examples of (36) in order to gain an understanding of the effects of multipath interference on the worst-case error probability. In Fig. 2, we plot (36) as a function of signal-to-interference ratio $1 / \sigma^{2}$ for various fixed values of the number $N$ of chips per bit and signal-to-noise ratio $1 / \gamma^{2} \equiv E_{\mathrm{b}} / N_{0}$, labeled SNR. We observe, as expected, that we get better worst-case performance with increasing $N$ (this applies for all the subsequent plots). We also observe that the error probabilities hit a noise floor due to the presence of fixed AWGN in the channel.

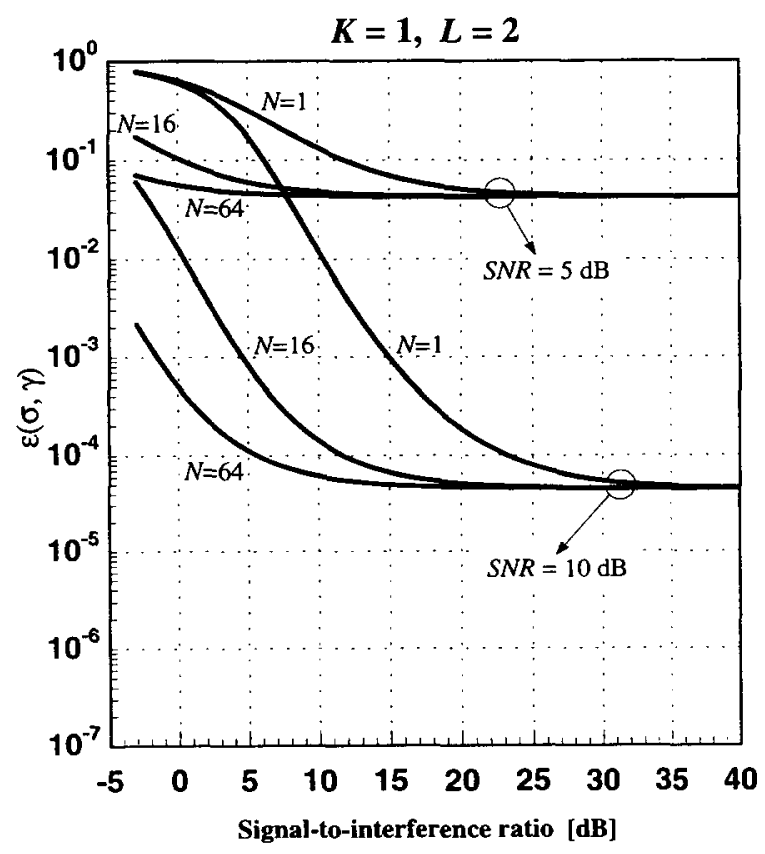

Fig. 2. Worst-case multipath performance: SIR varies. 
In Fig. 3, we plot (36) as a function of signal-to-noise ratio $E_{\mathrm{b}} / N_{0}$ for various fixed values of $N$ and signal-to-interference ratio $1 / \sigma^{2}$, labeled SIR. Here, we also observe the noise floor phenomenon, this time due to the presence of fixed multipath interference. As expected, the noise floor moves up as $1 / \sigma^{2}$ is decreased. In contrast to Fig. 2 , however, the noise floor also moves up as $N$ is decreased. This is due to the fact that the worst-case error probability is a function of $N$ in a pure multipath channel, whereas it is independent of $N$ in a pure AWGN channel. We further see that the worst-case performance for small values of $N($ e.g. $N=1)$ is very poor, whereas for large values, it is very good. This result verifies the utility of direct-sequence spreadspectrum in combating multipath interference.

In order to directly compare the effects of multipath interference to those of AWGN, we define the composite signal-to-noise ratio as

$$
\mathrm{SNR}_{c} \triangleq r \frac{1}{\gamma^{2}}+(1-r) \frac{1}{\sigma^{2}}, \quad 0 \leqslant r \leqslant 1 .
$$

Hence, as $r$ varies from 0 to 1 , the channel changes from a pure multipath interference channel to a pure AWGN channel while the total noise power remains fixed for a fixed $S_{N R}$ value. In Fig. 4, we plot (36) as a function of the composite signal-tonoise ratio $\mathrm{SNR}_{\mathrm{c}}$, for various fixed values of $N$ and $r$. We observe the zero-error region phenomenon when we have a pure multipath channel with no AWGN $(r=0)$. Since the worst-case is defined with respect to a constraint on the signal-to-interference

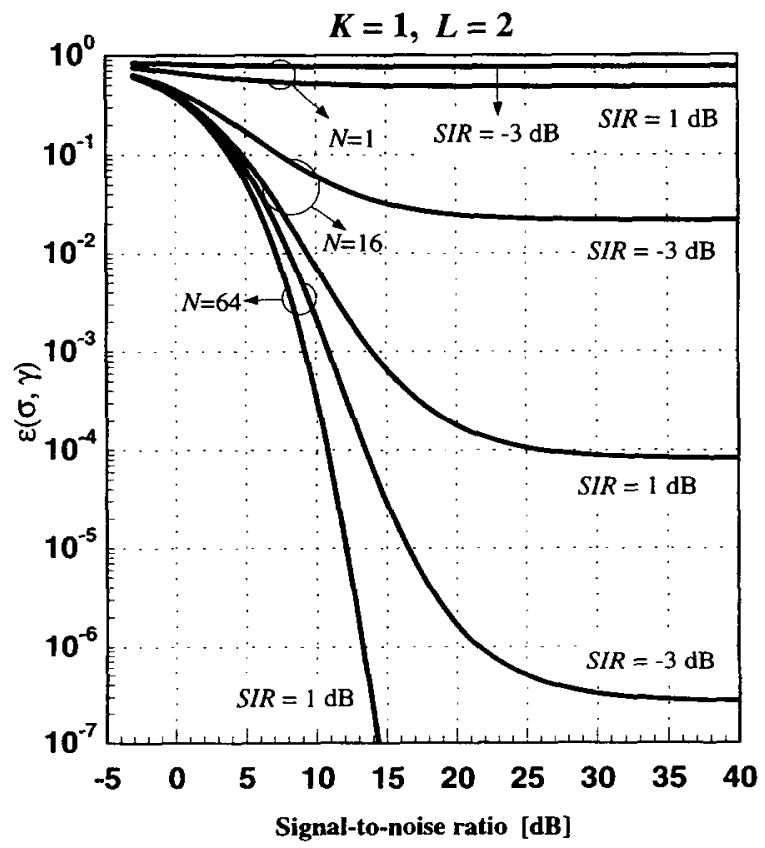

Fig. 3. Worst-case multipath performance: SNR varies. 


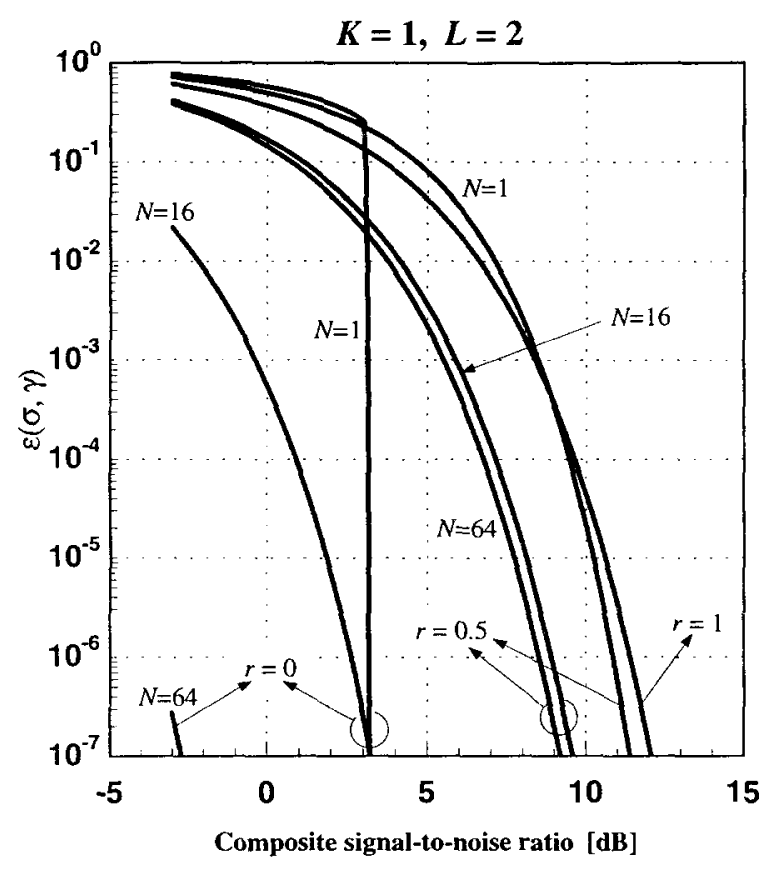

Fig. 4. Worst-case multipath performance: $\mathrm{SNR}_{\mathrm{c}}$ varies.

ratio, the zero-error region emerges for signal-to-interference ratios greater than a certain value, as the only term contributing to the error is due to the multipath interference. Indeed, an investigation of (21) reveals that if $1 / \sigma^{2}>2(K L-1)$, there can be no error in the absence of $\tilde{\eta}$, since $X_{k, l} \leqslant N$ and $Y_{k, l} \leqslant N$. In our case, this corresponds to $1 / \sigma^{2}>2 \equiv 3.01 \mathrm{~dB}$. Hence, $r=0$ curves sharply fall to zero for $1 / \sigma^{2}>3.01 \mathrm{~dB}$. We also see from Fig. 4 that as $r$ is increased, the channel becomes more and more AWGN-like. In fact, at $r=1$, we get the basic pure AWGN channel. In this case, we get the same performance for all $N$, since the number of chips per symbol is irrelevant in a pure AWGN channel, direct-sequence spread-spectrum corresponds to BPSK, and the worst-case analysis becomes irrelevant.

\subsection{Effects of multiuser interference}

In order to gain an understanding of the effects of multipath interference on the worst-case error probability, we plot several examples of (37) with $K=2$ and $L=1$. Figures $5-7$ correspond to replacing multipath interference with multiuser interference in Figs 2-4.

We see that all the comments and observations made for the multipath plots apply exactly the same way for the multiuser plots. In fact, we observe through a comparison of the two sets of plots that the difference in worst-case performance between the two 


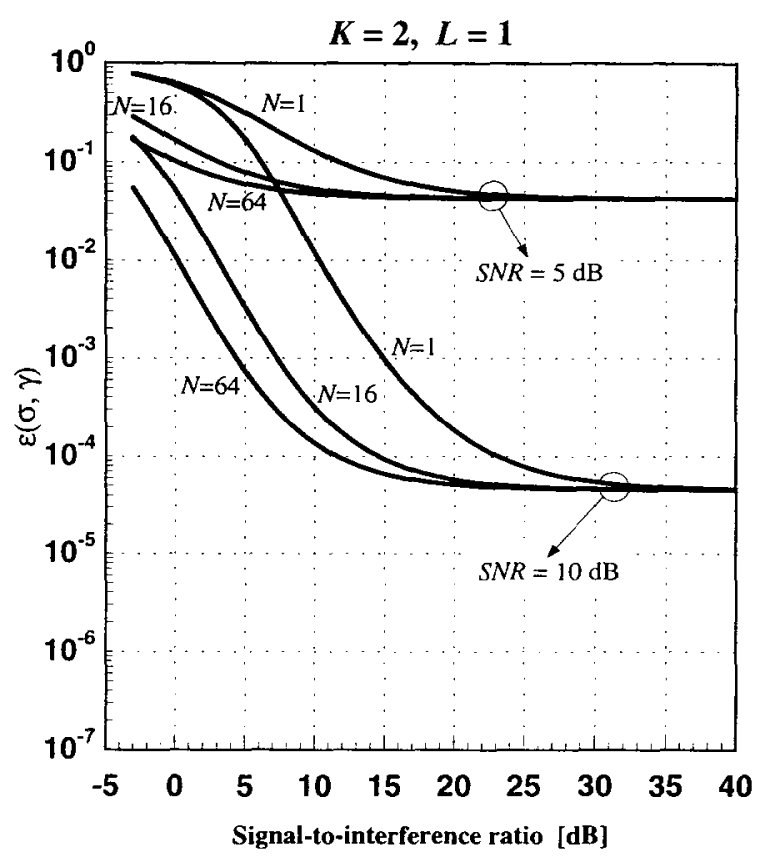

Fig. 5. Worst-case multiaccess performance: SIR varies.

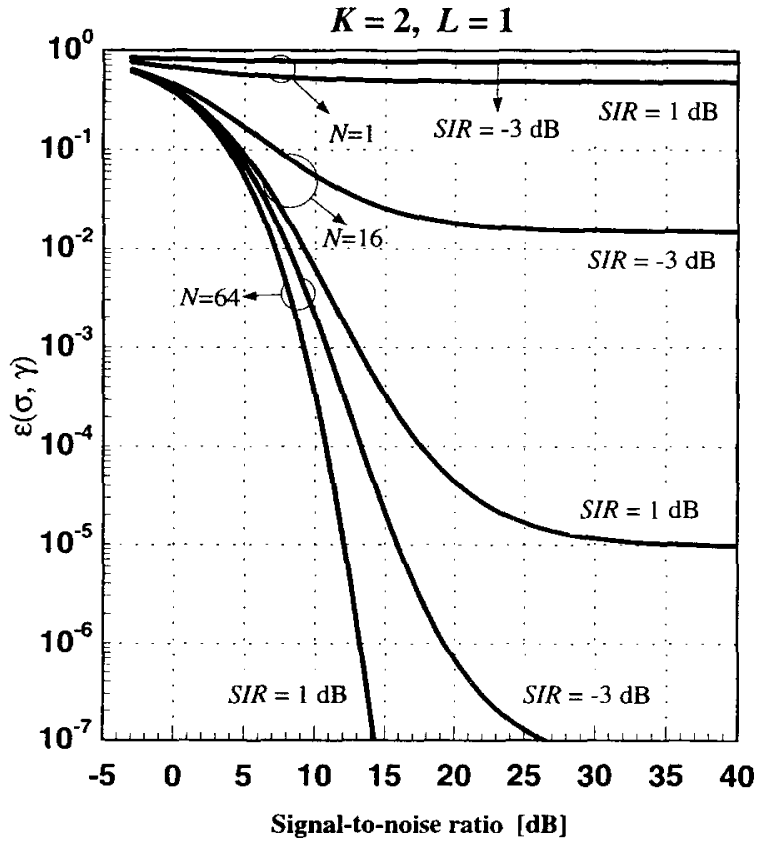

Fig. 6. Worst-case multiaccess performance: SNR varies. 


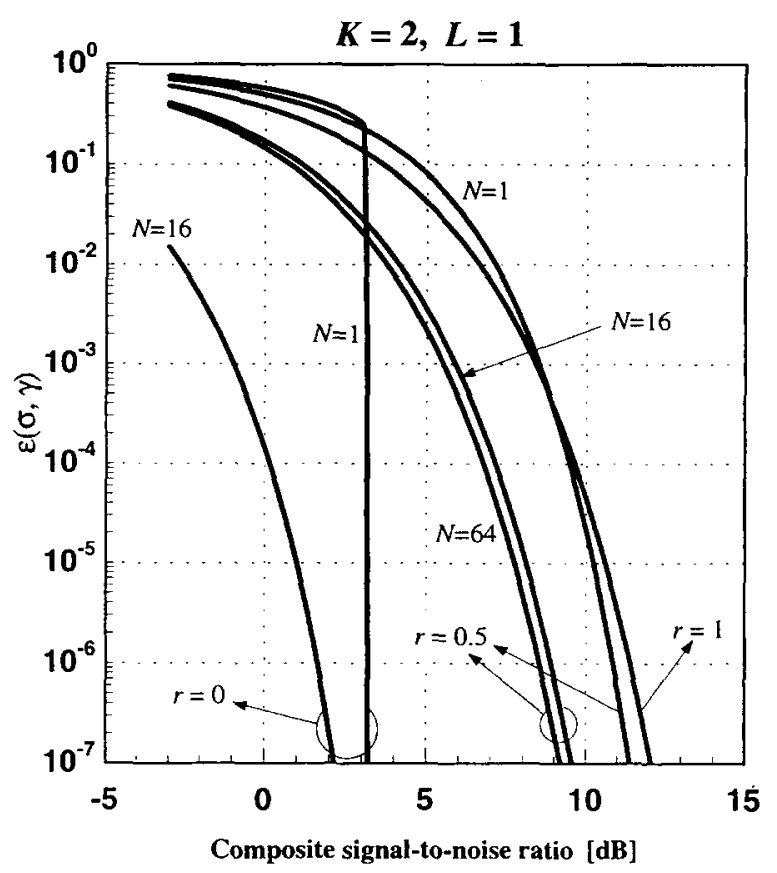

Fig. 7. Worst-case multiaccess performance: $\mathrm{SNR}_{\mathrm{c}}$ varies.

cases is very small, almost imperceptible except for Fig. 6 versus Fig. 3, where the difference is more obvious, especially in terms of the noise floors. In any case, the multipath worst-case performance appears to be slightly worse than the multiuser worst-case performance. When the fraction of AWGN in the channel is increased, however, the difference all but disappears.

\subsection{Conclusions}

We have seen through several examples that the worst-case performance of a directsequence spread-spectrum system in a multipath or multiuser environment can be very poor for low values of $N$, but is remarkably good for high values of $N$. One immediate conclusion is the superior interference rejection capability of directsequence spread-spectrum, whether the interference is due to multipath or multiuser.

We have also observed that the difference in the worst-case performance between a multipath channel and a multiuser channel of equivalent interference power is not significant, although the multipath performance is very slightly worse. This shows that a correlation between the received signal and interference makes things worse, but only slightly.

Since the performances in both cases are very similar, we can generalize our conclusions to the case where we have $K$ transmitters with $L$ paths each. The difference in performance between having $K L$ transmitters with one path each and one trans- 
mitter with $K L$ paths is expected to be relatively small. And the general case, $K$ transmitters with $L$ paths, will lie in between the two.

\section{References}

[1] M. Kavehrad, P.J. Mclane, Spread spectrum for indoor digital radio, IEEE Commun. Mag. 25 (6) (1987) 32-40.

[2] M. Kavehrad, B. Ramamurthi, Direct-sequence spread spectrum with DPSK modulation and diversity for indoor wireless communications, IEEE Trans. Commun. COM 35 (2) (1987) 224-236.

[3] M. Kavehrad, P.J. MeLane, Performance of low-complexity channel coding and diversity for spread spectrum in indoor, wireless communication, AT\&T Tech. J. 64 (8) (1985) 1927-1965.

[4] K. Pahlavan, M. Chase, Spread-spectrum multiple-access performance of orthogonal codes for indoor radio communications, IEEE Trans. Commun. 38 (5) (1990) 574-577.

[5] R. Ganesh, K. Pahlavan, Statistics of short time and spatial variations measured in wideband indoor radio channels, IEE Proc. H 140 (4) (1993) 297-302.

[6] S.J. Howard, K. Pahlavan, Autoregressive modeling of wide-band indoor radio propagation, IEEE Trans. Commun. 40 (9) (1992) 1540-1552.

[7] M.K. Simon, J.K. Omura, R.A. Scholtz, B.K. Levitt, Spread Spectrum Communications Vol. I. Computer Science Press, Rockville, Maryland, 1985

[8] J.G. Proakis, Digital Communications, McGraw-Hill, 1983.

[9] L. Xuedong, A worst-case analysis of direct sequence spread spectrum in indoor wireless channels, M.S.E.E. thesis, Cleveland State University, Cleveland, OH, 1995.

[10] H. Stark, J.W. Woods, Probability, Random Processes, and Estimation Theory For Engineers, 2nd edn. Prentice Hall, 1994.

[11] M. Hizlan, B. Hughes, Worst-case performance of asynchronous spread-spectrum multiple-access. IEE Proc. Commun., submitted.

Post-print standardized by MSL Academic Endeavors, the imprint of the Michael Schwartz Library at Cleveland State University, 2014 